\title{
KNIFELESS MANAGEMENT OF EARLY ECTOPIC PREGNANCY
}

\begin{abstract}
Mousumi Dutta ${ }^{1}$, Nibedita Chattopadhyay², Sandhya Das ${ }^{3}$, Dipankar Gupta4 ${ }^{4}$ Gourango Sarkar 5 , Arghya Maitra ${ }^{6}$, Ibadahun Suchiang ${ }^{7}$, Rajsekhar Saha ${ }^{8}$
\end{abstract}

${ }^{1}$ Assistant Professor, Department of Gynaecology and Obstetrics, CNMC, Kolkata.

${ }^{2}$ Associate Professor, Department of Gynaecology and Obstetrics, CNMC, Kolkata.

${ }^{3}$ Assistant Professor, Department of Gynaecology and Obstetrics, CNMC, Kolkata.

${ }^{4}$ Associate Professor, Department of Paediatrics, SSKM and H, Kolkata.

${ }^{5}$ Assistant Professor, Department of General Medicine, SSKM and H, Kolkata.

${ }^{6}$ Associate Professor, Department of Gynaecology and Obstetrics, CNMC, Kolkata.

7 Ist Year Postgraduate Trainee, Department of Gynaecology and Obstetrics, CNMC, Kolkata.

${ }^{8}$ Assistant Professor, Department of Gynaecology and Obstetrics, Berhampore Medical College and Hospital, Murshidabad.

ABSTRACT

\section{BACKGROUND}

Ectopic pregnancy (EP) always demands surgical management. But with advent of modern diagnostic approaches with serum $B$ HCG with high resolution TVS, now it is possible to diagnose early EP. Methotrexate (Mtx) is a promising drug to manage these patients conservatively and results are equivalent to surgery. We have shown it in our study.

\section{MATERIALS AND METHODS}

Uncontrolled clinical trial of 63 cases of medical management of EP was undertaken. These 63 patients fulfilled the selection criteria and rest were excluded from our study. Demographic data, clinical presentation and treatment outcome were captured. All patients were managed indoor and side effect of the treatment and treatment failures were identified early by followup and then managed.

\section{RESULTS}

From January 2013 to April 2016, 63 patients with EP were successfully treated with intramuscular Mtx, only 4 patients required laparotomy. No major side effects were noted, but mild pain in abdomen. Their fertility outcomes were also preserved.

\section{CONCLUSION}

Systematic Mtx injection is safer and alternative to surgical procedure of selective early EP. But prior criteria must be fulfilled and constant followup is needed to identify failure cases and complications.

\section{KEYWORDS}

Methotrexate, Ectopic Pregnancy, Serum Beta HCG, Transvaginal Sonography [TVS], Laparotomy, Fertility.

HOW TO CITE THIS ARTICLE: Dutta M, Chattopadhyay N, Das S, et al. Knifeless management of early ectopic pregnancy. J. Evolution Med. Dent. Sci. 2017;6(85):5897-5899, DOI: 10.14260/jemds/2017/1282

\section{BACKGROUND}

Ectopic pregnancy is an acute emergency condition if not diagnosed and intervened on time. It can reduce maternal morbidity and mortality in a greater way if earlier diagnosis and appropriate treatment starts. Its incidence is $1.5 \%-2 \%{ }^{1}$ In pregnancies from ART, it rises from $3 \%-5 \% .^{2}$ Though surgical management remains the mainstay of treatment, laparoscopy is gold standard currently.

With the availability of high resolution TVS and expedient Sr. ß-hCG assay at most early pregnancy assessment with EP now-a-days is diagnosed much earlier and in a more benign nature.

Systemic Mtx was first recognised as medical treatment for unruptured EP by Tanaka et al in 1982,3 and since then it is popularised as a safe and effective medical management alternative to surgery.

'Financial or Other Competing Interest': None.

Submission 01-08-2017, Peer Review 07-10-2017,

Acceptance 14-10-2017, Published 23-10-2017.

Corresponding Author:

Dr. Mousumi Dutta,

\#11/23, Kali Charan Ghosh Road,

Sinthi More, Kolkata - 700050.

E-mail:drmdms@gmail.com

DOI: $10.14260 /$ jemds $/ 2017 / 1282$
Currently, three main treatment regimens exist. Single dose protocol, 2 doses protocol and multi-doses protocol depending upon trends of Sr. B-hCG level and time for resolution of EP.

As single dose regimen was introduced to minimise the side effect, to improve patient's compliance and to reduce cost, our study aimed to give single dose treatment and if needed 2 doses regimen and their outcome.

But we did careful followup and assessment for all women before assuming that the treatment has failed and required surgical intervention.

\section{MATERIALS AND METHODS}

An uncontrolled clinical trial was conducted among ectopic pregnancy patients admitted in gynaecology ward of Calcutta National Medical College and Hospital in between January 2013 and April 2016. Only 63 ectopics fulfilled the selection criteria and rest were excluded. After approval from local ethical committee and taking prior informed consent from the patients, we started to recruit the cases based upon our selection criteria. Patients who were haemodynamically stable with $\mathrm{Sr}$. ß-hCG $</=5000 \mathrm{mlU} / \mathrm{mL}$, adnexal mass $>2-3$ $\mathrm{cm}$, absent cardiac activity and presence of haemoperitoneum $<100 \mathrm{~mL}$. The patients with haemoperitoneum > $100 \mathrm{~mL}$, adnexal mass $>3 \mathrm{~cm}$ and presence of cardiac activity and $\mathrm{Sr}$. 
ß-hCG > $5000 \mathrm{mlU} / \mathrm{mL}$ were excluded from our study. Baseline investigations such as full blood count, Sr. ß-hCG, RFT, LFT and blood group were done and on $\mathrm{D}_{1}$ single dose of $50 \mathrm{mg} / \mathrm{m}^{2}$ Mtx was administered. Serial Sr. ß-hCG was repeated on $\mathrm{D}_{4}$ and $\mathrm{D}_{7}$. If Sr. $\beta$-hCG on $\mathrm{D}_{7}$ was at least $15 \%$ lower than on $\mathrm{D}_{4}$, the patient was discharged and followed up as an outpatient until Sr. ß-hCG was $<5 \mathrm{mlU} / \mathrm{mL}$. If Sr. ß-hCG was higher or same as $D_{4}$, the patient received a $2^{\text {nd }}$ dose of $50 \mathrm{mg} / \mathrm{m}^{2} \mathrm{Mtx}$. Again, follow-up the patient with Sr. ß-hCG weekly until $<5 \mathrm{mIU} / \mathrm{mL}$ or any complication developed; $2^{\text {nd }}$ dose Mtx for both persisting plateauing of Sr. ß-hCG and increasing Sr. ß-hCG.

For patients with haemodynamic instability, signs of tubal rupture, increasing abdominal pain and falling haemoglobin demanded surgery.

\section{Statistical Analysis}

The collected data were checked, screened and coded before entering into the data sheet of software (SPSS 16.0). For descriptive statistics, mean, median, range, standard error and proportion were used. Confidence interval, odds ratio was calculated. Chi-square analysis was done for determining any association of statistical importance between treatment outcome and other related variables. ( $\mathrm{P}$ value of $<.05$ was considered significant).

\section{RESULTS}

Total no. of cases $(n=63)$. Among them 4 cases failed and laparotomy was done. Among others 14 patients delivered a live baby, 5 had infertility and 8 patients lost for followup; 7 cases were given double dose Inj. Mtx $\mathrm{D}_{4}$. In this study, the mean age was 29.70 ranging between $20-37$ years. It is found that the association between final outcome and age is insignificant. Success rate was maximum between (20 - 25) years' age group and thereafter it decreased as age increased. (Table 1) Gravidity is between 1 - 2. Nulliparous ( $n=41$ ) 65\%, primiparous ( $n=17) 27 \%$ and multipara $(n=5) 7.9 \%$. Highest success rate is seen among primiparous ladies (100\%) followed by nulliparous (92.7\%) and multiparous (80\%). Chisquare test shows that the p-value is .277 . So, the association between final outcome and parity is insignificant (Table 2). Total 4 patients underwent laparotomy for rupture of ectopic pregnancy. Success rate is high (98\%) if sac size is $<2 \mathrm{~cm}$ and it is decreased if sac size is $>2-3 \mathrm{~cm}$ (76.9\%). It is found that there is association between sac size and final outcome. It is significant $(\mathrm{p}=.024$, which is $<.05)$ (Table 3). Among 63 cases, 56 patients got single dose Mtx after checking pretreatment Sr. B-hCG repeated on D4 and D7. The other 7 patients got double dose Mtx due to non-satisfactory Sr. ßhCG value on D4 (either increasing or same value). The average value of Sr. ß-hCG on D1 of single dose Mtx was 1765 (range 550 - 4500) and treatment with double dose Mtx was 2064 (range 400 - 3500). On D7, the mean Sr. ß-hCG is 702 for single dose and 1017 for double dose Mtx. Total no. of cases was 63; 56 (88.9\%) cases were treated with single dose Mtx. Among them 53 responded well (94.6\%); 3 had rupture (5.4\%). Seven cases were given double dose Mtx and successful patients were $6(85.7 \%)$ and failed case $1(14.3 \%)$ (Table 4). Among 63 cases of Mtx treated, total no. of laparotomy were 4, delivery (ND/CS) 14, infertility 5 and 8; patients lost for followup after resolution of EP. Most of the patients have complained of lower abdominal pain after getting Mtx. Some complained of mild fever, nausea, vomiting and generalised body ache. Some patients had mild vaginal bleeding; 4 had laparotomy either due to increased lower abdominal pain, increased Sr. B-hCG value or USG diagnosed haemoperitoneum and vitals instability.

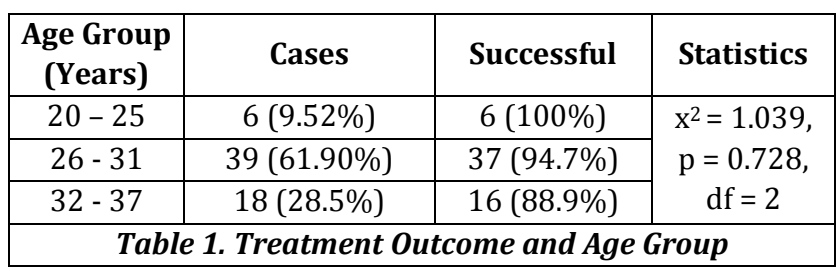

\begin{tabular}{|c|c|c|c|}
\hline Parity & Cases & Successful & Statistics \\
\cline { 1 - 3 } 0 & 41 & $38(92.7 \%)$ & $\mathrm{x} 2=2.784$ \\
\cline { 1 - 3 } & 17 & $17(100 \%)$ & 0.222 \\
$\mathrm{df}=2$
\end{tabular}

\begin{tabular}{|c|c|c|c|}
\hline $\begin{array}{l}\text { Adnexal Mass } \\
\text { (cm) }\end{array}$ & Cases & Successful & Statistics \\
\hline$<2$ & 50 & $49(98 \%)$ & \multirow{2}{*}{$\begin{array}{c}\mathrm{x} 2=7.708 \\
\mathrm{p}=0.024 \\
\mathrm{df}=1\end{array}$} \\
\hline$>2-3$ & 13 & $10(76.9 \%)$ & \\
\hline
\end{tabular}

\begin{tabular}{|c|c|c|c|}
\hline \multirow{2}{*}{$\begin{array}{c}\text { Number of } \\
\text { Methotrexate } \\
\text { Dose }\end{array}$} & \multicolumn{2}{|c|}{ Final Outcome } & \multirow{2}{*}{ Statistics } \\
\hline & Unsuccessful & Successful & \\
\hline Single dose & $3(5.4 \%)$ & $53(94.6 \%)$ & \multirow{2}{*}{$\begin{array}{c}x^{2}=83 \\
p=0.38 \\
d f=1\end{array}$} \\
\hline Double dose & $1(14.3 \%)$ & $6(85.7 \%)$ & \\
\hline
\end{tabular}

Table 4. Number of Dose vs. Treatment Outcome

\section{DISCUSSION}

Mtx therapy for EP of all routes and doses combined has a success rate of between $74 \%-84 \%^{1}$. The success rate tends to be higher if selection criteria is strictly maintained. Kooi and Koch noted a success rate of $94 \%$ when initial Sr. B-hCG level was less than $10,000 \mathrm{IU}$ as compared to $68 \%$ when the initial Sr. ß-hCG level was above 10,000 IU. ${ }^{2}$

Lipscomb et al also concurred with respective rates of $94 \%$ and $75 \%{ }^{3}$. Stika et al found that patients on Initial Sr. ßhCG of above 5000 IU had a greater probability of requiring either multiple doses of Mtx or surgery for their ectopic gestation. ${ }^{4}$ In our study, we selected patients whose Sr. B-hCG were $<5000$ IU. Because Stroel and Liing 5 and Lipscomb et $\mathrm{al}^{3}$ found that the presence of cardiac activity within the ectopic pregnancy reduced the success rate from 95\% to $70 \%$. We excluded ectopic pregnancies with a positive cardiac activity.

With respect to the gestational sac size, Shaleu et al found a success rate of $76 \%$ when conceptus was smaller than $2 \mathrm{~cm}$ and $52 \%$ when it was lesser than $2 \mathrm{~cm}^{6}$.

In our study, we found $83.1 \%$ success rate with Mtx when sac size $<2 \mathrm{~cm}$ and $25 \%$ failure $^{2}$ rate. Out of 50 patients who had $<2 \mathrm{~cm}$ sac size. We also have seen $16.9 \%$ success rate vs $75.0 \%$ failure rate when sac size $>2-3 \mathrm{~cm}$ out of 13 patients. 
So with increasing sac size decreases the success rate. Total 4 patients had laparotomy ( $6.3 \%$ ) out of 63 patients.

Srivichai et al ${ }^{7}$ reported a success rate of $90.6 \%$. In 96 out of 106 patients were successfully treated with Mtx, though 4 received a $2^{\text {nd }}$ dose. Success reached $90 \%(n=10)$ in patients out of 11 with single dose treatment in Merisio's series. ${ }^{8}$

In our study, we found 59 patients' treatment successfully with Mtx (93.7\%). Though 7 patients required double dose and $6.3 \%$ failed, 3 from single dose and 1 patient from double dose. We believe the high success rate of our study is due to careful selection of cases and confirming 3 strictly to the criteria of low Sr. ß-hCG levels adnexal mass $<3 \mathrm{~cm}$ and proper clinical assessment avoided unnecessary surgical intervention.

Mamodh demonstrated that Sr. ß-hCG of $2000 \mathrm{mlU} / \mathrm{mL}$ is an optimum cut-off value for selecting potential cases for medical failure using the single dose approach as cases with initial Sr. ß-hCG value of $>2000 \mathrm{mIU} / \mathrm{mL}$ or an embryonic sac size $>3.4 \mathrm{~cm}$ should be closely monitored for treatment failure. ${ }^{9}$ This correlates with our study because the mean pretreatment Sr. $\beta$-hCG is $1765(n=56)$ in our study, so that we get a high success rate (93.7\%). The mean Sr. B-hCG of double doses is $2064(n=7)$. In the same study, increased treatment failure is seen with increased age of patients and in our study, we get $100 \%$ success rate when age is between $20-25(n=6)$ and success rate is decreasing with increasing age that is $62.1 \%$ ( 26 - 31 yrs.) and $27.6 \%$ (32 - 32 yrs.).

The incidence of infertility as a risk factor reported in literature was $30 \%$ for EP. ${ }^{10}$ However, it accounted for $(n=5)$ infertility in our study. Though $n=8$, patients had missed followup. Total $n=63$, lost for followup 8,5 patients had infertility $(11.11 \%)$.

In our study, the women mainly complained of pain in the lower abdomen. It simulates with the feature of ruptured ectopic. The pain may be due to tubal abortion or directly due to drug itself $11 ; n=48$ patients complained of pain abdomen, but out of them 4 received laparotomy. No other complaints; $\mathrm{n}=2$ patients complained of mild vaginal bleeding. Future fertility outcome was followed. In one study following medical treatment of EP, conception was $66 \%$ and subsequent live birth was $30 \%{ }^{12}$ These results are comparable to our study where live birth is $n=14$ (31.1\%).

\section{CONCLUSION}

Mtx has proven to be an effective medical management for EP. It offers several benefits over surgical treatment. Less invasive, less expensive, better compliance and future fertility preserving benefits give us a first choice than surgery in early EP. However, either indoor patient management or on an outpatient basis close patient monitoring to be done with availability of TVS/TAS or Sr. ß-hCG. The predictors of success rate in our study are low Sr. $\beta$-hCG and adnexal mass $<3 \mathrm{~cm}$.

In conclusion, we found that success rate of our study is 93.5\% with 52 single dose and 7 double dose Mtx treatment with no severe morbidity. So conservative approach is superior than surgical approach.

\section{REFERENCES}

[1] Yao M, Tulandi T. Current status of surgical and nonsurgical management of ectopic pregnancy. Fertile Stetil 1997;67(3):421-33.

[2] Clasen K, Camus H, Tournaye H, et al. Ectopic pregnancy: let's cut! Strict laparoscopic approach to 194 consecutive cases and review of the literature on alternatives. Hum Reprod 1997;12(3):596-601.

[3] Kooi S, Kock HC. A review of the literature on nonsurgical treatment in tubal pregnancies. Obstet Gynecol Surv 1992;47(11):739-49.

[4] Lipscomb GH, McCord ML, Strovall TG, et al. Predictors of success of methotrexate treatment in women with tubal ectopic pregnancies. $\mathrm{N}$ Engl J Med 1999;341(26):1974-8.

[5] Stika CS, Anderson L, Frederiksen MC. Single-dose methotrexate for treatment of ectopic pregnancy: Northwestern Memorial Hospital three-year experience. Am J Obstet Gynecol 1996;174(6):1840-8.

[6] Stovall TG, Ling FW. Single dose methotrexate: an expanded clinical trial. Am J Obstet Gynecol 1993;168(6 Pt 1):1759-65.

[7] Shalev E, Peleg D, Bustan M, et al. Limited role for intratubal methotrexate treatment of ectopic pregnancy. Fertil Stetil 1995;63(1):20-4.

[8] Srivichai K, Uttavichai C, Tongsong T. Medical treatment of ectopic pregnancy: a ten-year review of 106 cases at Maharaj Nakorn Chiang Mai Hospital. J Med Assoc Thai 2006;89(10):1567-71.

[9] Merisio C, Anfuso S, Berretta R, et al. Single-dose methotrexate for Ectopic pregnancy treatment: preliminary data. Acta Biomed 2005;76(1):33-6.

[10] Mamdoh E. Single dose methotrexate for treatment of ectopic pregnancy: risk factors for treatment failure. Middle East Fertility Society Journal 2007;12(1):5762.

[11] Zargar M, Razi T, Barati M. Comparison of single and multidose of methotrexate in medical treatment of ectopic pregnancy. Pak J Med Sci 2008;24(4):586-9.

[12] Barnhart KT, Gosman G, Ashby R, et al. The medical management of ectopic pregnancy: a meta-analysis comparing "single dose" and "multiple dose" regimens. Obstet \& Gynecol 2003;101(4):778-84. 